\title{
Is Logic in the Mind or in the World? Why a Philosophical Question can Affect the Understanding of Intelligence
}

\author{
Hanns Sommer \\ Lothar Schreiber \\ Universität Kassel, Fachbereich 15 \\ Mönchebergstr. 7 \\ D-34119 Kassel
}

Editor: Kai-Uwe Kühnberger

\author{
HANNS.SOMMER@MRT.UNI-KASSEL.DE \\ LSCH@IFM.MASCHINENBAU.UNI-KASSEL.DE
}

\begin{abstract}
Dreyfus' call 'to make artificial intelligence (AI) more Heideggerian' echoes Heidegger's affirmation that pure calculations produce no 'intelligence' (Dreyfus, 2007). But what exactly is it that AI needs more than mathematics? The question in the title gives rise to a reexamination of the basic principles of cognition in Husserl's Phenomenology. Using Husserl's Phenomenological Method, a formalization of these principles is presented that provides the principal idea of cognition, and as a consequence, a 'natural logic'. Only in a second step, mathematics is obtained from this natural logic by abstraction.

The limitations of pure reasoning are demonstrated for fundamental considerations (Hilbert's 'finite Einstellung') as well as for the task of solving practical problems. Principles will be presented for the design of general intelligent systems, which make use of a natural logic.
\end{abstract}

Keywords: Cognition, Natural logic, Intelligence, Husserl, Hilbert.

\section{An Engineer's Look at Grounding Problems}

On which principles can 'intelligence' be based? To find an answer to this question, we may look at the grounding of our knowledge, and at our ability of making 'intelligent' decisions.

Grounding problems are considered by many scientists to be well posed only within the framework of a theory, and therefore those problems are treated as being purely theoretical problems. On the other hand, practical problems in engineering, in AI, and in robotics have demonstrated the need to understand the meaning of 'intelligence' in general (Wang, 2007, 2009). Trying this will change the focus: instead of proving the completeness or consistency of a theory, the main interest will be to make systems 'more intelligent'.

As logics are thought to be the most important tools of 'intelligent behavior', one has to ask for their provenance as Gila Sher has done (Sher, 2010): 'Is logic grounded in formal or structural laws governing the world?' or 'does sensory experience play a central (if not exclusive) role in logic?', shortly 'Is logic in the mind or in the world?'

Though Sher's question seems to be quite theoretical, the more so it was discussed in a philosophical journal, the question has important practical consequences for artificial general intelligence (AGI). To make artificial systems intelligent, it is generally accepted to use heuristic algorithms, which consist of mathematical parts (e.g. the definition of a search space with a 
neighborhood structure and an objected function), but also employ some strategies, like 'greedy search' strategy, that are justified only by experiences of whom ever. So, these non-mathematical parts are alway doubtable. It remains obscure where they come from and how, in general, we are able to find them. Their adequacy and optimality never can be ascertained in advance, and their formulations tend to be nebulous.

The main idea of our paper is based on Husserl's distinction between 'a logic of truth' on one side, and on the other side 'mathematical logics' understood as "Idealwissenschaft" (Hartimo, 2011). Husserl considers his 'phenomenological method' to be a first philosophy, or a first access to cognition taking place before any logic is available.

A a result of this, some statements by means of cognition are made true, and these true beliefs are called knowledge. This definition corresponds to the epistemic view that 'knowledge essentially is representation' (Tarrazo, 2004). But, as is explained with reference to Brentano's and Husserl's idea of 'intention', true beliefs need not be specified by complicated processes in the knower's brain. The structures of knowledge obtained thus provide a 'logic of truths'.

Following some of Husserl's ideas, and reformulating them in Fuzzy Logic with Belief Theory, we deduce a knowledge from principles of cognition and empirical data only. The examination of this 'natural knowledge' provides a logic which, in correspondence to the work of P. Maddy (Maddy, 2007), we call 'natural logic'.

We find that natural logic always is context-dependent, and therefore incomparable with mathematical set-theory and any other mathematical theory based on it. Natural logic is not very convenient for calculations, but it has one important property: it contains no other structures than can be identified in the empirical data. In that sense natural logic is minimal.

In contrast, if a problem is solved with purely mathematical methods, the problem must be modeled completely within mathematical theories by means fixed in advance. Therefore the means may be considerably more complex than the problem itself, and will make the problem intractable. This is why a theory of AGI must combine mathematical methods with cognition methods. The objective of the present paper is to explain the differences, but also the common origin of these methods.

In section 2 a formal access to a natural logic is presented. Some philosophical statements are cited to support our understanding of some of Husserl's introductory texts and the ideas we derived from them. We don't aim at a systematic discussion of Husserl's thinking in its historical context.

In section 3 the relation between a natural logic and a mathematical logic is examined. It will be shown by examples that a mathematical logic may create structures that cannot be confirmed by cognition. At the end of this section we contrast our understanding of 'knowledge' to Platon's theory of ideas, and discuss the meaning of knowledge.

In section 4 hints are given, how to use a natural logic to solve real practical problems, and we discuss for which type of problems these concepts will be adequate.

Section 5 considers the relation between our work and the actual discussion in AGI about the meaning of logic. We observe strong relations between AGI and physics.

The central role of cognition is summarized in the conclusion.

\section{Intelligence as the ability of cognition}

The way an engineer looks at problems he has to solve provides a practical definition: 
A system or an agent are said to be intelligent if they are capable to select from a set of actions, they can carry out, the most effective one to solve the current problem of a set of problems posed to them.

Practical intelligence therefore is not an absolute capability. It depends on:

- the set of problems to be solved,

- the environment in which the agent has to solve the problems,

- the set of actions, which the agent has at his disposal,

- the estimation of the success of actions on former problems and the memory of it,

- the sequence, in which the problems are offered to the agent.

Intelligence is strongly intertwined with the old philosophical problem of cognition, about which many philosophers have pondered and sought for a foundation of it (Chandrasekharan, 2009). Cognition must be comprehended in its general sense: besides the objects of our world, it has to provide the concepts of reasoning.

Edmund Husserl invented the 'Phenomenological Method' to deduce concepts from the experiences we obtain in our natural life-world. This method enables us to transcend subjective knowledge into an objective understanding. Husserl claims that our complete knowledge, our subjective perceptions, and all concepts are based on our life-world. Essential concepts are deduced from the 'horizon' given by the subjective structure by removing (or 'putting into parenthesis', in Husserl's words) from a complexity of thoughts everything that can be taken away without destroying the meaning. This procedure is called the 'Phenomenological Reduction Method'.

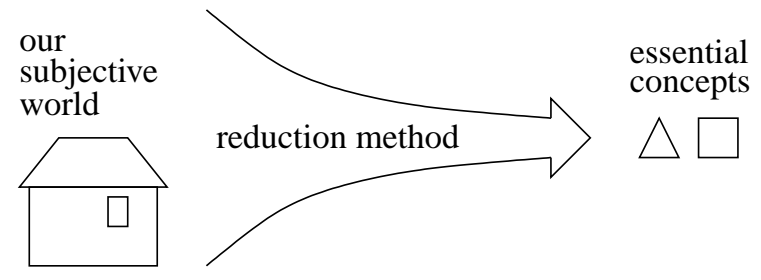

Using the phenomenological method we can see cognition as a process by which meaning is deduced from empirical data relative to intentions.

As our knowledge, thus obtained, is qualified by our intentions, great part of it is subjective, that is to say, depending on ourselves. But there are also intentions that can be fixed objectively, and respective to these intentions, all beings, sharing the same data, will build the same concepts. (An example for intentions of that kind is presented in section 2.2.) Husserl's Phenomenological Method provides an unambiguous determination of a cognition process. Every cognition, and with it, every knowledge in phenomenology is based on Husserl's Principle of all Principles (Husserl, 1913; Hopp, 2009):

'Every originary presentive intuition is a legitimating source of knowledge ... everything originarily ... offered to us in "intuition" is to be accepted simply as what it is, presented as being, but also within the limits, in which it is presented there ... This "vast world" appears as finite, as a "constitutive result" that is, with the sense that 
its intelligibility is a product of intentional acts and intuitive presentations that have gradually constituted it through time. What the world means for us, is ultimatively dependent on our own limited subjectivity [sic].'

A perception will be constructed with a meaning-constituting activity (noesis) in a process we call cognition. This process may be very complex, but taking Husserl's phenomenological method into consideration, the essential idea of cognition, on its lowest level as a simple apprehension, can be reduced to basic concepts. All results of this paper are based on the following postulate:

The data, the recognising agent's relation to these data, and his intentions (represented by the questions for which he searches answers) each constitute a concept necessary in every cognition.

Based on this postulate, it is claimed that the cognition process presented in section 2.2 reduces to a minimum the presuppositions and preconception for cognition.

Remark: This result of minimality is our motivation to include some of Husserl's ideas into an interdisciplinary discussion of the basic tools for AGI. Besides simple apprehensions, there also exist higher levels of comprehension, which correlate objects of consciousness to more complex intentions, which are systematically discussed in a recent philosophical paper of Kosowski (Kosowski, 2012).

The definitions presented in sections 2.1 and 3, precisely: knowledge, natural logic, and mathematical formalisms, are believed by us to compare to some aspects of Husserl's ideas: 'Anschauung', 'Sprache', and 'Kalkül' (Zuh, 2011).

\subsection{Derivation of a natural logic from operational principles}

In a first step (First Philosophy), Husserl deduces the principal concepts characterising some idea of our life-world by the 'transcendental-phenomenological reduction'. These concepts become the tool for all further steps (Second Philosophy (Maddy, 2007)). So, to capture the essential idea of cognition the following concepts are needed (Schreiber and Sommer, 2010):

I Empirical data: Here empirical data is represented by measurement tuples:

$m_{1,1}, m_{1,2}, \ldots, m_{1, k_{1}}, m_{2,1}, m_{2,2}, \ldots, m_{2, k_{2}}, m_{3,1}, m_{3,2}, \ldots$

II The relation to this empirical data: Empirical data may appear to us as big, medium, small, tiny, or having some other property.

Formally this relation is represented by Fuzzy predicates $P$, describing the degree, up to which a measurement value $m_{i, j}$ satisfies a property.

For technical reasons, all values will be represented here by rational or real numbers. The value $Z_{P}(m) \in[-1 ; 1]$ of the membership-function $Z_{P}$ can be interpreted as the strength of our feeling that the value $m$ belongs to the predicate $P . Z_{P}(m)$ near 1 indicates strong agreement, and $Z_{P}(m)$ near -1 strong refusal.

The predicate $\neg P$ is represented by the membership-function $-Z_{P}(m)$. 


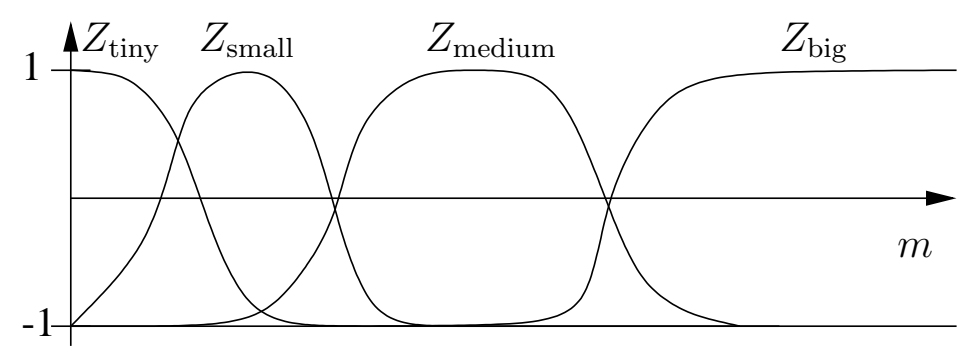

III The intention to obtain knowledge: This is formalised in two steps:

- Confirmation of sentences: Sentences $A_{i}$ are formulated with predicates and andoperators, for example: ' $m_{j, 2}$ is great and $m_{j, 7}$ is not small and $m_{j, 3}$ is small'.

A statement with an or-operator like ' $m_{1,2}$ is $P_{1}$ or $m_{1,7}$ is $P_{2}$ '

can be rewritten with an and-operator: ' $\operatorname{not}\left(m_{1,2}\right.$ is $\neg P_{1}$ and $m_{1,7}$ is $\left.\neg P_{2}\right)$ '.

The confirmation of a sentence will be obtained from the membership degrees $d_{i, j}:=$ $Z_{P_{i}}\left(m_{j, i}\right)$ of its $n$ 'atoms' $\left(m_{j, i}\right.$ is $\left.P_{i}\right)$ for $k$ measurement tuples $m_{j, i}, \quad(i=$ $1, \ldots, n ; j=1, \ldots, k)$ with an aggregation operator Agg : $\bigcup_{i=1}^{\infty}[-1 ; 1]^{i} \rightarrow[-1 ; 1]$ by the expression (see appendix A):

$$
\operatorname{Agg}\left(d_{1,1}, d_{2,1}, d_{3,1}, \ldots, d_{n, 1}, d_{1,2}, d_{2,2}, \ldots, d_{n, 2}, d_{1,3}, \ldots, \ldots, d_{n, k}\right)
$$

- The process of knowledge acquisition: All knowledge depends on the agent's intentions that find their expression in the questions he asks his world.

A question is formed by a sentence $A$ and an agent, who asks for the believability of it relative to his empirical data.

The complexity of a sentence $A$ by definition is the length of the shortest text that represents $A$ in a binary alphabet $\{0 ; 1\}$. Fano's Theorem tells us that all the bits of this representation are independent and of equal importance. (This fact is a consequence of the minimality of a Fano code (Hutter, 2009b).)

Let $Z(A)$ denote the confirmation obtained for $A$ by expression (1) and $d$ the unknown confirmation of a bit in the shortest bit-text for $A$ with length $C(A)$. As the bits of the text must provide the confirmation for $A$, we obtain the equation (2), which specifies a function $F_{C(A)}(d)$ :

$$
Z(A)=\operatorname{Agg}(\underbrace{d ; d ; \ldots ; d}_{C(A) \text { factors }})=: F_{C(A)}(d)
$$

This equation can be rewritten:

$$
d=F_{C(A)}^{-1}(Z(A))
$$

As the believability $\operatorname{BEL}(A)$ of a sentence $A$ should be equal to the confirmation of its description, equation (3) gives the result:

$$
\operatorname{BEL}(A)=F_{C(A)}^{-1}(Z(A))
$$

Strongly believable sentences constitute knowledge. 
By steps from I to III (natural) knowledge is obtained from empirical data and our relation regarding this data according to our intentions (question $A$ ?). For a sentence $A$, to be part of the knowledge, two conditions have to be satisfied:

I Question $A$ ? has to be asked.

II By steps I to III the inequality $\operatorname{BEL}(A) \gg \operatorname{BEL}(\neg A)$ is approved.

But the believability of a sentence is not independent from other previously posed questions, and the knowledge they provided. The believability $\operatorname{BEL}(\mathcal{A})$ for a set of sentences $\mathcal{A}$ is obtained as a whole by the same procedure used for a single sentence. The believability of every sentence $A \in \mathcal{A}$ depends on $\mathcal{A}$.

Respective to the context $\mathcal{A}$, a sentence $A$ belongs to the knowledge, if the following two conditions are satisfied:

I Question $A$ ? has been asked.

II By steps I to III the inequality $\operatorname{BEL}(\mathcal{A} \cup\{A\}) \gg \operatorname{BEL}(\mathcal{A} \cup\{\neg A\})$ is approved.

The context-dependency of our natural knowledge has been explicitly stressed by Husserl. He writes (Barber, 2009):

'These complex syntheses [of knowledge] cannot remain isolated. All particular syntheses through which things in perception, in memory are given, are surrounded by a general milieu and they do not float there in an isolated manner, but rather, are themselves synthetically intertwined with one another.'

Acceptable imaginations are only those that can be obtained as knowledge from a real world. Inference rules are rules that produce new sentences from sets of believable sentences. An inference rule is called admissible respective to a knowledge, if it transforms strongly believable sentences of that knowledge into strongly believable sentences. Thus, the knowledge constitutes the data for the calculation of the degree of believability of interference rules. (To make this definition unambiguous, it would be necessary to fix the degree, wherefrom strong believability begins.)

Natural logic is constituted by sets of strongly believable sentences (axioms) and corresponding admissible inference rules. As the believability of the result of the application of an inference rule to a set of sentences is obtained by an aggregation of the respective believabilities of the rule and the sentences, the believability of this result is always less than (or as an exception equal to) that of the sentences. Therefore, long sequences of conclusions can not result in highly believable results. Because of the impossibility to build an unlimited sequence of inference rules in natural logic, the confirmed knowledge will always be finite.

Summary: (I) An observer's basic knowledge is obtained from empirical data, always with regard to his intentions. This knowledge is constituted by a set of sentences and by their respective degrees of believability. The believability of one single sentence depends on the whole knowledge. Believability therefore is a context-dependent property.

(II) As basic knowledge, again, is empirical data, it provides the possibility for the constitution of higher order knowledge: knowledge about knowledge.

Concepts are defined by sets of sentences and the membership degrees up to which they 
correspond to a certain concept. Any property of a concept is represented by a sentence which strongly corresponds to that concept. Any imagination consists of knowledge that can be constructed with admissible inference rules from believable knowledge. The transmission from simple apprehensions to logical judging will be produced by further and more complex intentions, as e.g. the demand of tractability and consistency of knowledge, cf. section 3 .

\subsection{Objections of physicists}

Many physicists will not be satisfied with the results, we have obtained so far, because they are convinced of the objectivity of physical laws. But as we have seen, any natural logic is subjective, because it depends on the empirical data and on the observer's relation to this data, especially on his intentions. To obtain the objectivity of physical laws we have to remember the principal objective of physics. The physicists' intention may be summarised by the general motto (Sommer, 2009):

'Find a knowledge by which forecasts of future measurements are enabled best.'

Knowledge that has been constituted respectively to this intention depends only on the empirical data, which were obtained in an experiment. Context-dependency, on the other hand, characterises natural knowledge just the same way as it characterises the most basic physical theory (quantum mechanics): Carlo Rovelli, Grete Hermann, Jeeva Anandan and other physicists gave a relational, that is context-dependent interpretation for quantum mechanics, which eliminates the concepts of the absolute state of a system and the absolute value of physical quantities (Herzenberg, 2008; Stuckey, Silberstein, and Cifone, 2009). Relational theory only describes the ways systems affect each other.

Concepts of knowledge are called context-independent, if no context can be imagined, in which they lose their properties. Likewise, for physical systems we get the

Definition: A physical system $\mathrm{S}$ is defined by a preparation-process, that is by actions producing a structure satisfying some test measurements. A property $\mathrm{P}$ that can be directly ascribed to $\mathrm{S}$ alone so that the assumption of S really possessing P does not lead to contradiction with any measurement that can in principle be done on $\mathrm{S}$, is called a context-independent property of S. Properties that do not satisfy these requirements are called context-dependent properties of S (Hajicek and Tolar, 2009).

The context-independence of a property P of a system S depends on the whole system. It is obvious that very great systems which are defined by a huge set of measurements will not be changed significantly by a single new measurement, because the lot of measurements already known provides a large confirmation of knowledge that cannot be disturbed by a single new measurement.

The cognition process produces an adaptation between a system and its environment. Because of this, the environment requires redundant reports of the system S. Now, many observers can find out the state of $S$ independently and without perturbing it. This is how preferred states of S become objective (classical) (Zurek, 1998, 2007; Hornberger, 2009; Zeh, 1999; Schlosshauer, 2008; Sommer, 2009; Symons, 2008). This effect is called decoherence. So, classicality emerges from natural as well as from quantum mechanical knowledge by fishing for context-independent information.

A natural Logic is principally different from a mathematical logic. As natural logic is context-dependent, conclusions using self-reference (for example Cantor's diagonal method) are 
not allowed, because every conclusion increases the observer's knowledge and therefore changes the context.

The important point to be learned from these considerations is that a cognition process in general does not provide a classical ontology nor a reality, which is composed of independent objects. To obtain a mathematical or set-theoretical description from a recognised knowledge, abstractions are necessary.

\section{An introduction to mathematical logic, the ability to think abstractly}

Husserl means by formalisation: "To abstract from the matter by substituting "object variables" for the names of "materially determinate objects", (Stenlund, 2010). The properties of the objects are then specified by the axioms of the theory.

In this way, mathematical objects are supposed to be abstract entities (Batterman, 2009). They can be obtained from natural knowledge by abstraction:

- A set of highly believable sentences is selected.

- Some of the terms in the sentences are generalised by abstracting them from some features. (Hermann Weyl wrote (Weyl, 1968): 'The decisive step of mathematical abstraction [is]... to forget about what the symbols stand for.')

- The abstracted sentences are declared absolutely true and context-independent. They form the axioms of a mathematical theory.

- An inference rule is mathematically admissible, if no reality can be imagined, where this rule is not admissible.

Example 1: We start with the sentence:

'To a set of $n$ sheep one can always add one sheep to get $(n+1)$ sheep.'

By abstracting from the feature 'sheep', and assuming absolute truth, we obtain Peano's axiom: $n \in \mathcal{N} \Rightarrow(n+1) \in \mathcal{N}$.

As there is no conceivable exception from this axiom inside the conception an agent obtains from our world by natural logic, Peano's axiom is suitable for mathematics.

The here sketched path to a foundation of mathematics (e.g. Peano's axiom) is strongly related to the ideas of the famous mathematician David Hilbert (Hilbert, 1964). As there is no direct access to pure ideas (of an 'Idealwissenschaft), an 'Urdomain' and a 'finite Einstellung' are needed to justify the foundations of mathematical theories. Each true mathematical statement should be based on the following principles (Stenlund, 2010):

- 'All logical inferences have to be founded on a finite basis that depends on our faculty of representation.'

- 'Mathematical truth is obtained by proofs.'

- 'A formalised proof, like a numeral, is a concrete and survey-able object. It can be communicated from beginning to end.' 


\subsection{Gödel's Objections}

In Hilbert's view, mathematics is the theory of theories. But is a theory of theories really possible? Because of Kurt Gödel's results, many mathematicians mistrust Hilbert's notion. Gödel's understanding of mathematical concepts was deeply influenced by Plato. In Plato's view, mathematical concepts are pure ideas and the basic condition of any existence. Plato postulates that the ideas are first and reality is only their approximative realisation. Absolute, context-independent entities in this view are the building blocks of everything. Thus, in abstract mathematics, selfreference is admissible. Based on this philosophical position, and presupposing consistency and the existence of the set of theorems in the theory of natural numbers, Gödel was able to prove the existence of unprovable true theorems. This result suggests the insufficiency of Hilbert's 'finite Einstellung'. The controversy between Hilbert and Gödel leads back to the question:

Is mathematics, as we understand it today, the fundament of every consistent conception?

To answer this question, we present the following examples, which demonstrate that besides Gödel's view also Hilbert's view is admissible. Especially in AI and in applied mathematics, Hilbert's view seems to be more promising. This will be the subject of Section 4.

Example 2: Recognition of identical entities in an absolutely empty space:

Hannes Leitgeb and James Ladyman discussed the question (Leitgeb and Ladyman, 2008):

'Is it possible to recognise two identical entities in an otherwise absolutely empty space?'

Their answer is deduced in a Platonian way of argumentation:

Firstly, they note that the mathematical structure consisting of two identical entities in an empty space consists of a graph $G=\{K, E\}$, where the set of nodes $K$ is defined by the two entities and the set of edges $E$ is empty.

In a second step, they conclude the possibility to recognise two identical entities in empty space "because graph theory postulates it and we have every reason to believe that the basic principles of graph theory are coherent: because we can generate graphical templates that indicate so, and so forth.'

This answer, however, is not valid in Hilbert's and Husserl's epistemic view. As we are unable to distinguish between two identical entities in an empty space, in a world that consists of only two identical entities we have no access to the number 'two'. Only from two distinguishable entities (for example two different sheep) we can construct the number 'two' by abstracting from the differences between these entities. Button's arguments therefore hold in an epistemic view (Leitgeb and Ladyman, 2008):

I 'It is unclear how we could have epistemic access to primitive identity facts.'

II [We have to question] "the "objecthood" of entities the numerical diversity of which would be primitive while they were at the same time indistinguishable from each other in terms of properties or relations.' 
The example demonstrates that different philosophical positions may generate different conceptions. If in a Platonian attitude, abstract mathematics are presupposed, then tasks appear to be resolvable that are not resolvable in a logic and a knowledge that is only deduced from epistemic methods. Merlau Ponty, in his philosophical essays, criticised the confinement of science to abstract mathematics giving exactly the same reason (Erdinast-Vulcan, 2007; Moran, 2008):

'Signification is always the articulation of separation, divergence or gap between figure and ground which is the enabling principle of the transition between the somatic and the semiotic.'

'Formalism's error is not that it overestimates form but that it esteems form so little that it abstracts it from meaning.'

The next example demonstrates that cognition may produce knowledge, which is incompatible with basic mathematical results.

Example 3: Weierstrass' continuous function, which is nowhere differentiable:

As velocity is a concept of our empirical experience for some curves $x(t)$, the meaning of differentiability exists also in our natural knowledge and in our empirical reality. In the $19^{\text {th }}$ century many mathematicians believed that every continuous function is piecewise differentiable. But Weierstrass gave an example showing the falsity of this belief. We demonstrate with the following Lemma that such a function cannot be the result of a behaviour of any system that has been obtained from empirically observed discrete measurements.

The example demonstrates that the mathematical concepts of correctness and consistency of an idea are not enough to make the 'existence' of that idea in a real world believable. We learn from Husserl that in a real world both, a logic of truths (or, in our words, a natural logic), as well as mathematical logics are needed. Therefore AGI as an engineering science is based on these two kinds of logic.

Lemma: Let $f(x)=y$ denote the most believable function describing the measurements $\left\{\left(x_{i}, y_{i}\right) ; i=1,2, \ldots n\right\}$, then from $f(x)$ is continuous follows that $f(x)$ is piecewise differentiable.

Proof: The proof of the Lemma is given in appendix B.

'Knowledge' is a controversially discussed idea in philosophy. Our definition of 'natural knowledge' is more specific than others, but has some restrictions, too. As a consequence of contextdependency, natural logic does not allow self referential conclusions, so, Platon's absolute ideas are not available (Schreiber and Sommer, 2010).

This is not the only shortcoming, as some very instructive comments of one reviewer demonstrate:

- 'The entire conception under consideration (in section 2) seems to depend on the degree wherefrom strong believability begins. It seems questionable whether such a definition can be made without arbitrariness.'

- 'Assume all measurements have been taken within a certain partial area of a domain. A new measurement is conducted in a new area outsides the initial one (but still within the same 
overall domain), and suddenly the rules and laws allegedly governing the entire domain show to be only at work within the first limited area, but have to be adapted (e.g. generalized) to also cover the second area, and thus the entire domain. What now?'

- 'What about the classical idea of "refutation by a single counterexample"?'

The first comment demonstrates that believability strongly depends on the context, in which statements are considered. For example, if we ask for the clemency of the weather planning a walk, the degree, wherefrom strong believability begins, can be chosen to be considerably lower than the degree we would apply asking for the stability of a bridge. Because of this context-dependency, the theoretical formalism by itself cannot fix the degree, from where strong believability begins.

The second observation of the reviewer stresses that natural knowledge does not generally hold outside the sub-domain it was obtained in. Our laws are dependent on the context, in which they were found. Even the knowledge obtained from a surrounding domain does not contain the complete knowledge of the sub-domain. This fact, well-known in quantum-mechanics, can be elucidated by a simple figure.
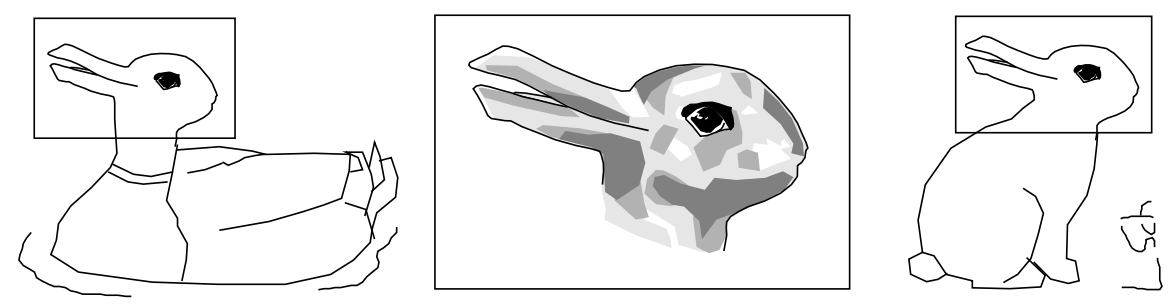

The left figure shows a duck, the right one a rabbit. In the middle, the sub-domain can be seen that is contained in both outside figures. It shows the well-known 'duck-or-rabbit' problem, confirming the believability of both sentences: 'This is a duck's head' and 'It's a rabbit's head'. The believability of the first sentence is completely lost in the right picture, that of the second is lost in the left one.

To the question last listed there is the definite answer 'No' - singular events cannot lead to true beliefs. In physics and engineering an experiment has to be repeatable to provide knowledge.

Knowledge depends on the science, by which it is deduced, and by which it is applied. Ayer says (Ayer, 1956): 'To have knowledge is to have the power to give a successful performance.' As we are interested in engineering practice, we have to show in section 4 that natural and mathematical logics are suitable to engineering problems.

\section{Intelligence of Technical Systems}

Every engineering task deals with the problems of a present state (which is hopefully precisely known) and some desired future objectives, namely solving the problems and possibly all other problems arising on the way. Although the task of solving problems is rewarding in itself, any solver of problems finds reward and esteem from former sufferers. The engineer's way of solving problems is to build technical devices and equipment, which are bound to become more and more adaptable to serve today's ever changing conditions and restrictions. The mechanical-, electro-technical- and computer-scientific parts of a device called 'machine' can no longer be designed separately by disciplines that ignore each other, but have to be intelligent and learning, see also (Froese and 
Ziemke, 2009). As for technical systems, the rewards are defined by external authorities, their learning capabilities and intelligence can be measured by their efficiency in reinforcement learning problems, ref. (Hutter, 2009a).

However, in these considerations, the most important question for AI does not become visible:

Do the tools, which machines or agents can use to solve reinforcement learning problems, constitute a mathematically well defined set?

Giving an affirmative answer to this question, opens a discussion of the meaning of 'intelligence' in the framework of the mathematical theory of complexity (founded by Kolmogoroff and Solomonoff). Many authors take part in this discussion (Hutter, 2009a; Legg and Hutter, 2007; Veness et al., 2009; Hernandez-Grallo and Dowe, 2010). Veness, Ng, Hutter and Silver have presented principal requirements of these argumentations. Discussing engineering tasks, they define:

'The general reinforcement learning problem is to construct an agent that, over time, collects as much reward as possible in this setting [that is the given task].'

To obtain a mathematically treatable problem, they give the following specification of the environment of an optimal agent, called 'AIXI':

'The AIXI setup mirrors that of the general reinforcement problem, however the environment is assumed to be an unknown but computable function, that is the observations and rewards received by the agent, given its actions can be computed by a Turing machine.'

An ideal agent reacts in accordance with the following rules (Veness et al., 2009):

- 'The agent considers the sum of the total reward over all possible sequences of future actions up to a fixed number of $m$ steps ahead.'

- 'It weights each of these rewards with the complexity of programs that calculate this sequence of actions on an universal Turing machine and whose calculations are consistent with the agent's past. The sum of these weighted rewards constitutes an expected reward.'

- 'The agent picks the action that maximises expected future rewards.'

As most practical problems are intractable or NP-difficult, it is normally not possible to realize an ideal agent. The presented principles only define, what is optimal behaviour of an agent, if complexity is not an issue. Veness et al. therefore make 'an attempt to scale AIXI down to produce a practical agent that can perform well in a wide range of different, unknown and potentially noisy environments.' The ideal agent is their means to explain, what artificial intelligence is.

But this explanation is problematic for systems acting under the conditions of insufficient knowledge and limited resources, more precisely, artificial general intelligent systems. For example, the arduousness of assurance companies consists of those risks and uncertainties, which have no mean value and no variance, or cannot even be modelled by probability distributions. So, systems acting in real life have to take account of a negative answer to the main question above.

Giving a negative answer, we can discuss the idea of 'intelligence' no longer in a well-defined mathematical frame. Even if the two main questions for problem solving, namely: 
(1) What are we looking for? (Intentions)

(2) Where are we searching? (Clarification of the environment)

find mathematically formulated answers, they never reduce the solving process to a pure mathematical task. The reason for this conclusion has been presented in Section 3: The logic of a part of reality is not unambiguously determined by mathematics.

What can be learned from psychology, is the impossibility of scaling down, by mathematical means only, the solution principles used to design an ideal AIXI-agent in order to create a practical one:

'The agents' interpretation of stimuli, rewards and actions will be of crucial importance ... This dependence on interpretation does not fit immediately into a reinforcement learning model, conceived of mechanistically ... Sophisticated cognitive processing applies to the interpretation of stimuli, evaluation of rewards, and implementation of actions' (Chater, 2008).

The psychologist J.P. Broonen stresses that an intelligent agent has to transform its basic objectives into a form that allows their realisation in a real world. He describes this task as the process of the formation of an intention (Broonen, 2010):

'A cardinal distinction is to be made between forming a decision (i.e. an intention), primarily a motivational process and implementing it, a volitional process. Volition addresses the regulation of behaviour and environment after the intention has been formed. After a first motivational phase, during which the individual weighs up the cost and benefits of performing the behaviour which culminates with the development of a behavioural intention, a post-intentional, or volitional, phase is necessary in case of difficulties of reaching the goal during which the individual has to develop strategies and plans in order to ensure that her intention will be enacted in a third phase.'

Practical psychologists dissociate the original motivation of a human being (named 'will' by the philosophers Schelling and Schopenhauer) from its representation by behavioural processes and conscious goals formed to get them.

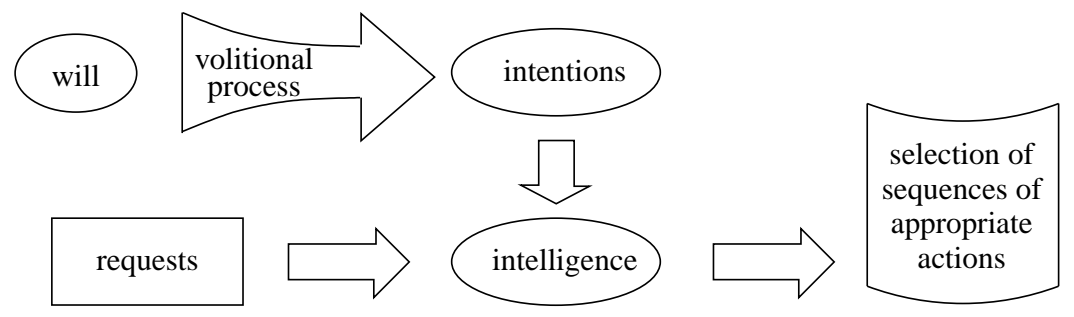

Will causes intentions, and intentions build intelligence such that requests are answered by the most adequate actions. In the language of engineers, this 'volitional process' translates into a very general and, at the first sight, very simple concept: the formation of interim goals. An interim goal is a goal that can be reached more easily and will serve as an orientation to reach the final goal. Nearly all methods used to make problems simpler can be interpreted as using interim goals:

- If a preparation step is necessary to solve a problem, the characterisation of this step constitutes an interim goal. 
- If a problem can be taken apart, the solution of its parts are interim goals that can be found more easily.

- A strategy to solve a problem can be understood as an interim goal that is formulated in a coarse graining language. For example each chess player knows the rule that to win a game, that is to conquer the opponent's king, it is recommendable to occupy first the central squares.

- A well known problem-solving-method is to simplify a problem by reducing the conditions under which the solution has to exist, to solve the simplified problem and to search for a modification of the solution to reach the final goal. The solution of the simplified problem is interpretable as an interim goal.

The difficulty to detect interim goals essentially depends on the language used to formulate the problem with all its requirements and requisites for its solution (Srivastava, Immerman, and Zilberstein, 2010). An optimal language to solve a problem in is the language of its interim goals. For finding the language, suitable for a solution plan, we recommend a procedure very similar to the algorithm of Section 2:

\subsection{A cumulative learning strategy to detect interim goals of a problem}

(a) Construct a set of similar and more simple problems to the original problem that has to be solved.

(b) A solution consists of a sequence of actions (a plan (Pollock, 2006)). Form sequences of actions by pure chance and

(c) test them for the set of problems defined in part (a).

(d) With the methods provided in section 2, find significant sentences that distinguish between successful and unsuccessful solution processes. The sentences which characterise the successful solution processes are suitable interim goals.

This methodology allows for focusing on interim goals and thus to simplify the search for an adequate solution language. Carrying out this search without taking the full complexity of the problems into consideration, is the same basic idea as found in many heuristics. But in designing heuristics, a guide is needed to find the path between Skylla and Charybdis. On one side, an incomplete language makes the problems unsolvable, whereas in most cases an exact and complete mathematical description will produce unsolvable complications. To navigate between these two poles, it is necessary to be aware of the restrictions of mathematical tools, and to master a method for the deduction of an adequate solution logic.

\subsection{Applications}

Problem solving may be interpreted as the task to learn a solution for a problem from the problem. Contrary, however, to the mostly probability-based learning techniques, AGI has to take more general views. E.g. in economy, agents are confronted with processes which are governed by power law distributions that have neither well defined mean values nor variances, and sometimes they have to deal with risks that have no probability distribution at all. In quantum mechanics, it is well known 
that new measurements may diminish the observer's knowledge and thus forbid an explanation of the phenomena in a Bayesian style. Human errors demonstrate that humans do not act logically in every situation, and it is supposed that this feat has survived with the fittest individuals along evolution. In this sense, illogical action sometimes seems to be the more effective strategy.

The methods presented in this paper are of interest wherever classical logic produces absurd or even contradictory results. The following example is well known in psychology (Tversky and Kahnemann, 1983), but it must be mentioned that our interpretation differs.

Example 4: Two statements (I) and (II) are presented to arbitrarily selected persons:

I $\underbrace{\text { Eulalia is a journalist. }}_{A}$

II $\underbrace{\text { Eulalia is a journalist }}_{A}$ with $\underbrace{\text { interest in politics }}_{B}$ and $\underbrace{\text { she is a member of a feminist society. }}_{C}$.

It comes out that more persons find statement (II) more believable than statement (I). This observation is not understandable from the view of classic logic and Bayesian probability theory, because for probabilities the inequality $p(A)>p(A$ and $B$ and $C)$ always holds.

However, respective to a knowledge structure obtained by means of section (2.1), the combination of the parts $A, B$, and $C$ of statement (II) produces an augmented believability for the average person, if one takes into account the believability of concepts of concepts (also mentioned in the summary (II) of the same section). In other words, the statements $B$ and $C$ produce a context, in which $A$ is more believable than without this supplementary information. Our explication correspond to the 'pragmatic paradigm' for the understanding of language in psychology (Canestri, 2012).

Even in engineering research, scientists more and more are forced to combine knowledge from very different planes, and have to cope with the problem that the results from one theory do not have a well defined meaning in the other theory. E.g., if fracture mechanics does combine Cauchy's continuum mechanics with quantum mechanical models of atomic structures, a 'heuristic bridge' is needed, which cannot be designed by physical means only. Generally, such 'heuristic' translations will construct the assignment between elements from different theories by regarding the similarity of the effects they produce. The similarity is specified and evaluated with methods from AGI.

In this way, both, engineering research and physics, need AGI not only for an evaluation of their models, but also for the modelling itself and for theoretical considerations (Kuusela et al., 2011). Very often the main problem to be solved when designing engineering tools, is to find formal terms for ideas that have been obtained from observations in practice. E.g. the onset of fracture of tools is a concept in practical engineering that is hard to translate into mathematics. A computer program that puts the cumulative learning strategy in practice to find fundamental formal terms for technical modelling was developed by the second author (Dürrbaum, Scherm, and Sommer, 2008).

Example 5: Forecast of drill fracture

It is always a difficult problem to fix the time for drilling processes when a drill had better be changed. If changed too early, good expensive drills may be wasted, if not, the broken tool may cause excessive damage to a valuable workpiece. In the actual technical application, measurements of axial force $F(t)$ and moment of torque $M(t)$ were available of always the same drilling process repeated with one and the same drill until fracture. The method of forecasting drill fracture consists of four steps: 
I An a priori given list of mathematical operations $\mathcal{O}_{i}$, each resulting scalar values $w_{i}$ when applied to a short sequence of numbers, is sequentially applied to the data of the first drilling process of a still undamaged drill, thus giving sets $\mathcal{S}_{i}$ of numbers $w_{i}$. The distributions of values in these sets are taken as guidelines to define fuzzy membershipfunctions $Z_{i j}\left(w_{i}\right)$ so that the values $w_{i}$ most clearly belong to the predicates $\mathcal{P}_{i j}$ chosen.

II The time axis also is divided in initially equal time slots $T_{k}$ which are mapped by fuzzy membership-functions $Z_{k}(t)$ to predicates $\mathcal{T}_{k}$ describing each time slot $T_{k}$. The parameters of $Z_{k}(t)$ are shifted in an ensuing optimization process to enhance the contrast of the mapping of values $w_{i}(t)$ to predicates $\mathcal{P}_{i j}$ of magnitude, as well as of time $\mathcal{T}_{k}$.

Now, the believability of the fuzzy sentence $S_{k i j}=\left(Z_{k}(t)\right.$ and $\left.Z_{i j}\left(w_{i}\right)\right)$ means: the value $w_{i}$ from operation $\mathcal{O}_{i}$ applied to data from a measurement channel for a time value $t$ satisfying time predicate $\mathcal{T}_{k}$, correspond strongly to predicate $\mathcal{P}_{i j}$. The believability $\mathcal{B}_{k i j}$ of sentence $S_{k i j}$ is calculated by the aggregation of all values of $S_{k i j}$ obtained for time values satisfying $\mathcal{T}_{k}$.

III The believability of all sentences $S_{k i j}$ is examined for all following measurement files with the aim to located the relevant sentences, i.e. those which keep the same believability in all measurements, but the very last few before fracture, where their believability changes drastically.

IV From the relevant sentences the final fracture criterion is derived by their aggregation.

The criterion found can be used in the industrial process, if the axial force and moment of torque are measured and can be processed online, to indicate to the operator the need to change the drill.

\section{The role of logic for artificial general intelligence}

If, in case of AI, intelligence may simply be understood as the ability to solve problems, the same definition will not be sufficient for general intelligence, as Look, Goertzel, and Pennachin stress (Goertzel, B. and Pennachin, C., 2007): 'A general intelligence must be able to carry out a variety of different tasks in a variety of different context.'

Such general objectives nearly always are affected by frame problems, like, in the history of sciences, they were extensively discussed in physics: Kant considered time and space as frames for every cognition, making both preconditions of physics. When Einstein demonstrated the dependence of space and time on matter distributions, the $19^{\text {th }}$ century physicists switched to causality being the unalterable principle of physics, but again the rise of quantum mechanics has cast new doubts on this conception (Mittelstaedt, 2011).

In the same way as physics needs a fixed description frame to determine the meaning of measurements, any reasoning system needs structures or at least some fragmentary logic enabling structured reasoning processes. If researchers in AGI could not be sure of the assumption that basically every intelligent process may be seen as a structured reasoning process mirroring a certain logic, it would be difficult to say, what AGI is researching.

On the other hand, any fixed structure or logic may conflict with the structures of some problems, thus complicating their solution. A general reasoning system therefore must be based on a logic that 
assumes only minimal structures for knowledge processes, or on mechanisms, which may not be recognized as logic any more. This, for example, is the case in Pei Wang's reasoning system (NARS) (Wang, 1994, 2011), which uses a non monotonic term logic with an inherence relation and bases the credibility of the inherence relatedness between terms on observations.

The 'logic' used in NARS is non-axiomatic, meaning no axioms are postulated. It is nonmonotonic, because new observations can overwrite prior judgments, and this 'logic' captures the principles of adaption to insufficient knowledge and resources. There seems to be no system with lesser needs of a priori specifications than NARS to capture information from a flux of observations in order to solve problems.

The knowledge processing system presented by us has many similarities to NARS, due to the common purpose to be as free as possible from prejudices. But, as our objective is more limited, there also are important differences. Our interest is guided by the question: 'Which are the basic principles that enable answers to questions respective to intentions and to a set of observations?'

Many difficulties that must be considered in the design of an intelligent reasoning system, such as restrictions of time, memory or hardware, or exigencies arising from communication between agents, are excluded from our considerations. In our understanding 'question answering' is the archetype of problem solving. In our real world we find problems entangled, but like physicists always seeking effects in most simple experiments, we try to recognize the foundational principles for problem solving in an universal but well-defined initial situation.

The function of the description language and the corresponding logic is not only to describe the world, but also to enable reasoning processes, an idea stressed by Danielle Macbeth: 'The good mathematical notion [in our wording description language] serves not merely to record something but to embody the reasoning, to put the reasoning itself before our eyes' (Macbeth, 2011).

Though artificial systems do not necessarily have eyes, the statement, nevertheless, applies to our context: The description language of an AGI system not only has to describe all relevant entities, but also has to make visible the solution obtaining concepts and algorithms. This second objective, corresponding to the frame problems in physics, cannot be achieved by mathematics itself, but it firstly needs some grounding principles, before mathematical or logical considerations can take place.

In the same way as physicists have searched for and found principles (energy conservation, least action principle, and so on) that hold in every frame, even if these frames are obtained from incompatible theories (Newton mechanics, general relativity, quantum mechanics, or quantum field theories) we are searching for principles of artificial general intelligence that are independent of logic.

Once these principles are known, they provide tools for the design of AGI systems. Firstly, an AGI system has to select from general principles those corresponding to its demands, and only in a second step it can specify a logic correspondingly to the chosen principles. Obviously, in an imprecisely known environment terms must be flexible and logical structures cannot be fixed in advance, whereas a simpler structure, as found in most games, allows the application of a mathematical logic.

Like physicists, who design their experiments in order to detect the principles of physics, in Section 2 we have designed a task to detect the cognition principles of AGI. Our most important result concerning the role of logic in AGI is the observation that mathematics as well as mathematical logics by themselves are insufficient tools, though they are often used, as Pennachin 
and Goertzel state: 'One approach of creating AGI is to formalize the problem mathematically and then seek a solution using the tools of abstract mathematics.'

But this approach can never exploit all the possibilities of AGI. As the deduction of knowledge by basic principles of cognition only provides context-dependent knowledge, neither the definition of terms, nor that of concepts can be founded solely on set theory or any other mathematical theory with context-independent entities.

Taking into consideration the difficulties of using context-dependent logics, it may be necessary to approximate the context-dependent knowledge with a context-independent language. But such a further step (see. Venesse et al.) is dependent on computing and action facilities, and therefore must be treated as an additional problem.

In an overall view, AGI must necessarily be seen as an embedded and embodied intelligence, and an intelligence like this cannot be limited to a self-contained mind, like pure mathematics can. Logic is the most effective tool the pure mind can use, but the adaption of logic to the world needs cognition. So, cognitive skills come primary to logic. The logic of an AGI system cannot be justified by rational reasoning only, but strongly needs empirical evidence. Wittgenstein (Philosophische Untersuchungen) has stated: 'An accordance in definitions is impossible without an accordance in the judgments.' That means that the definitions needed for the specification of the logic in an intelligent system must depend on our judgment of 'what is good'.

\section{Conclusions}

Clarifying the meaning of 'intelligence' is a very important objective for engineering projects in AI and robotics. As AGI systems act in open environments, they cannot a priori be equipped with a predetermined language conforming to the meaning of intelligence in all conditions of employment.

The discussion in this paper is based on the most fundamental feat an 'intelligent' system should achieve: The cognition of solutions to a problem.

The fundamental conditions of cognition, therefore, constitute the minimal requisites of every and even the most simple intelligent system. An examination of these conditions shows that 'intelligence' is an idea that cannot be obtained by classical ontology, but only in the context of the whole including the 'intelligent' actor, because 'intelligence' depends on the actor's view on the whole. An intelligent actor may have a 'free will' (as claimed by some philosophers), but an artificial system always corresponds to its creator's will, as well as the goals and subgoals used in the design of intelligent systems emerge from the creator's view, his intentions, and his desires.

The intelligence of an artificial system corresponds to two abilities of its creator:

1. To construct a language, or, in more precise terms, a logic, which corresponds to a given class of problems in the creator's own perspective. (Because of Quine's un-translatability-thesis, sometimes two or more languages will be necessary.)

2. To have sufficient command of these languages in order to find and compute solutions.

The second ability has a theoretical foundation (Hutter, 2009a; Legg and Hutter, 2007; HernandezGrallo and Dowe, 2010). But, as we have shown, the first ability escapes any mathematical formalism.

'Finding the "right" representation is a crucial problem' (Schmid and Kitzelmann, 2011), and a representation is a presupposition for a discussion of intelligence in mathematical terms. Quine's 
un-translatability-thesis causes the in-comparableness of intelligences based on different languages. As has been demonstrated in sections 3 and 4 the most intelligent view of some problems cannot be obtained from mathematical considerations only. 'Intelligence' has to be understood in relation to our real world, but not pertaining to formal theories obtained by abstractions.

Husserl's phenomenology starts with our real world. His Phenomenological Reduction provides cognition principles and, in succession, a natural logic. But the findings obtained from this natural logic do not correspond to the requirements of mathematical theories (for example: the contextindependent meaning of elements and operators). Cognition may present things more easily, and may exclude singular abstract mathematical objects. So, Heidegger's motto: 'Being true signifies to enable detections', leads to the astonishing observation:

Mathematics cannot constitute a complete foundation of AI, and, the other way around, AI is needed to determine the meaning of mathematical proofs, and with it, mathematical truth.

Having specified our questions and intentions, the principles of cognition provide a scientific foundation of justified belief, and thus a foundation of AI-methods within and beyond mathematics, as well as AGI methods.

Our discussion has disclosed the relation between intelligence and the capability of detecting knowledge. As these ideas cannot be disentangled, there does not exist a precise (mathematical) definition of intelligence, but only general principles, the realization of which creates intelligent behaviour. The detection and realization of these principles is the task of AGI. Our examples demonstrate that AGI's contribution is crucial to meet the challenges of modern engineering.

\section{Acknowledgments}

We would like to thank an anonymous reviewer for his extremely insightful and helpful comments.

\section{Appendix A: Aggregation Operators (Yager, 1994; Benferhat, 2010)}

Let $d_{1}, d_{2}, \ldots, d_{N} \in[-1,1]^{N}$ be a string of $N$ assessments. An aggregation operator transforms this string into one value $\operatorname{Agg}\left(d_{1}, d_{2}, \ldots, d_{N}\right) \in[-1,1]$ such that the following conditions hold:

- Equality: Each agent has the same influence.

- Commutativity: The value of an aggregation operator is independent of the order of the values $d_{1}, d_{2}, \ldots, d_{N}$.

- Monotony: If $d_{k} \leq \widehat{d}_{k}$ for $k=1, \ldots, N$ then $\operatorname{Agg}\left(d_{1}, d_{2}, \ldots, d_{N}\right) \leq \operatorname{Agg}\left(\widehat{d}_{1}, \widehat{d}_{2}, \ldots, \widehat{d}_{N}\right)$

- Neutrality: There exists a value $e \in[-1,1]$ such that

$$
\operatorname{Agg}\left(d_{1}, d_{2}, \ldots, d_{N}\right)=\operatorname{Agg}\left(d_{1}, d_{2}, \ldots, d_{N}, e\right) .
$$

- Associativity: $\operatorname{Agg}\left(d_{1}, d_{2}, \ldots, d_{k}, \operatorname{Agg}\left(d_{k+1}, \ldots, d_{N}\right)\right)=\operatorname{Agg}\left(d_{1}, d_{2}, \ldots, d_{N}\right)$.

- Accumulation: $\operatorname{Agg}\left(d_{1}, d_{2}, \ldots, d_{N}\right)<$

$$
\operatorname{Agg}\left(d_{1}, d_{2}, \ldots, d_{N}, d\right) \text { for } d=\max \left\{d_{1}, d_{2}, \ldots, d_{N}\right\}>e \text {. }
$$


It is interesting that this very general definition strongly determines the representation of any aggregation operator.

Representation Theorem Any aggregation operator Agg can be represented by the operators max, or min, or by the operators $\vee$ and $\wedge$ if the assessments have been rescaled by $\psi_{\vee}[-1, e] \rightarrow[0,1]$ and $\psi_{\wedge}[e, 1] \rightarrow[0,1]$

$$
((a \wedge b)=a \cdot b, \quad(a \vee b)=1-(1-a) \cdot(1-b) \text { for } a, b \in[0,1]) .
$$

The operator Agg is represented by the equations:

$$
\begin{aligned}
& \operatorname{Agg}\left(d_{1}, d_{2}\right)=\psi_{\wedge}^{-1}\left(\psi_{\wedge}\left(d_{1}\right) \wedge \psi_{\wedge}\left(d_{2}\right)\right) \text { for } d_{1}, d_{2} \in[-1, e], \text { and } \\
& \operatorname{Agg}\left(d_{1}, d_{2}\right)=\psi_{\vee}^{-1}\left(\psi_{\vee}\left(d_{1}\right) \vee \psi_{\vee}\left(d_{2}\right)\right) \text { for } d_{1}, d_{2} \in[e, 1]
\end{aligned}
$$

If a confirmation $d_{1} \in[e, 1]$ meets a negation $d_{2} \in[-1, e]$ there are various possibilities:

- Strong veto: Cancel any influence of confirmation $d_{1}$

- Optimistic evaluation: Cancel any influence of negation $d_{2}$

- Balanced decision: $\operatorname{Agg}\left(d_{1}, d_{2}\right)=\sqrt{d_{1} \cdot d_{2}}$

\section{Appendix B: Proof of the Lemma in Section 3}

Proof: The proof of the Lemma will be given in a generally accepted mathematical language. For any $x, f(x)$ is defined by the most believable value $y$ respective to the knowledge $\left\{\left(x_{1}, y_{1} \mid i=1, \ldots, n\right\}\right.$. The function $f(x)$ can be defined by the statement:

The more similar $x$ is to $x_{i}$ the more similar is $y$ to $y_{i}$

We define similarity with a distance measure $d\left(x, x_{i}\right)$ and a twice differentiable membershipfunction $Z$ : $\quad Z{ }_{d}$ and use the aggregation operator and $(=\wedge$ ). (That is to say assessments $d_{1}, d_{2} \in[0,1], e=1$, and $\operatorname{Agg}\left(d_{1}, d_{2}\right)=d_{1} \wedge d_{2}=d_{1} \cdot d_{2}$.)

Replacing $\left[Z\left(d\left(x, x_{i}\right)\right) \Rightarrow Z\left(d\left(y, y_{i}\right)\right)\right]$ by $\left[\operatorname{not} Z\left(d\left(x, x_{i}\right)\right) \vee Z\left(d\left(y, y_{i}\right)\right)\right]$, (5) can be reformulated with the equation:

$$
\begin{aligned}
y=\arg \underbrace{\max }_{y} \bigwedge_{i=1}^{n} \operatorname{not} Z\left(d\left(x, x_{i}\right)\right) \vee Z\left(d\left(y, y_{i}\right)\right)= \\
\arg \underbrace{\max }_{y} \underbrace{\prod_{i=1}^{n}\left(1-Z\left(d\left(x, x_{i}\right)\right)\right) \cdot Z\left(d\left(y, y_{i}\right)\right)}_{g(x, y)}
\end{aligned}
$$

As $f(x)=y$ is assumed to be continuous, $f(x+\triangle x)$ remains in a small neighbourhood of $f(x)$ and $y$ can be found by minimising $g(x, y)$ or by the equation:

$$
\frac{\mathrm{d} g(x, y)}{\mathrm{d} x}=0
$$

The implicit function theorem guarantees a differentiable solution of equation (6) for $\frac{\mathrm{d}^{2} g(x, y)}{\mathrm{d}^{2} x} \neq 0$. As this condition is satisfied almost everywhere for suitably defined functions $d$ and $Z$, we conclude that up to a finite set of discrete points, $f(x)=y$ is piecewise continuous. 


\section{References}

Ayer, A. J. 1956. The problem of knowlegde. Penguin Books, London.

Barber, M. 2009. Holism and Horizon: Husserl and McDowell on Non-conceptual Content. Husserl Stud. 25:1-14. doi: 10.1007/s10743-008-9035-5.

Batterman, R. 2009. On the Explanatory Role of Mathematics in Empirical Science. British J. Philosophy of Science 1-25. doi: 10.1093/bjps/axp018.

Benferhat, S. 2010. Interventions and belief cange in possibilistic graphical models. Artificial Intelligence 174(2):177-189.

Broonen, J.-P. 2010. Volition and the Theora of Planned Behaviour: How to Fill the Gap Between Intention and Performance? In Dubois, D., ed., Computing Anticipatory Systems, volume 1303, 315-323. CASYS'09 Ninth International Conference.

Canestri, J. 2012. Sprache, Neurowissenschaft und Psychoanalyse. Forum Psychoanalyse. DOI:10.1007/s00451-011-0092-6.

Chandrasekharan, S. 2009. Building to Discover: A Common Coding Model. Cognitive Science 33:1059-1086.

Chater, N. 2008. Rational and Mechanistic Perspectives on Reinforcement Learning. Cognition. doi: 10.1016/j.cognition.2008.06.014.

Dreyfus, H. L. 2007. Why Heideggerian AI Failed and how Fixing it would Require making it more Heideggerian. Artificial Intelligenc 171(18):1137-1160.

Dürrbaum, A.; Scherm, W.; and Sommer, H. 2008. Systemidentifikation mittels Glaubenskalkül. Automatisierungstechnik 7:350-356.

Erdinast-Vulcan, D. 2007. That Which 'Has No Name in Philosophy': Merleau-Ponty and the Language of Literature. Hum. Stud. 30:395-409. doi: 10.1007/s10746-007-9069-2.

Froese, T., and Ziemke, T. 2009. Enactive Artificial Intelligence: Investigating the Systematic Organization of Life and Mind. Artificial Intelligence 173(3-4):466-500.

Goertzel, B., and Pennachin, C. 2007. Artificial general intelligence. New York: Springer.

Hajicek, P., and Tolar, J. 2009. Intrinsic Properties of Quantum Systems. Found. Phys. doi: 10.1007/s10701-009-9296-7.

Hartimo, M. 2011. Husserl's Pluralistic Phenomenology of Mathematics. Philosophia Mathematica. DOI:10.1093phimat/nkr032.

Hernandez-Grallo, J., and Dowe, D. 2010. Measuring Universal Intelligence: Towards an Anytime Intelligence Test. Artificial Intelligence. doi: 10.1016/j.artint.2010.09.006.

Herzenberg, C. 2008. Grete Hermann: An early contributor to Quantum Theory. arXiv: 0812.3986[quant-ph]. 
Hilbert, D. 1964. Hilbertiana, fünf Aufsätze. Darmstadt: Wissenschaftl. Buchgesellschaft.

Hopp, W. 2009. Phenomenology and Fallibility. Husserl Stud. 25:1-14.

Hornberger, K. 2009. Introduction to Decoherence Theory. Lect. Notes Phys. 768:223-278.

Husserl, E. 1913. Ideen zu einer reinen Phänomenologie und einer phänomenologischen Philosophie. Hamburg: Meiner.

Hutter, M. 2009a. Open Problems in Universal Induction and Intelligence. arXiv: 0907.0746v1[cs.AI]4July2009.

Hutter, M. 2009b. Feature Reinforcement Learning: Part I. Unstructured MDPs. J. Artificial General Intelligence 1:3-24.

Kosowski, L. 2012. The structure of noema in the process of objectivation. Husserl Stud. doi: 10.1007/s10743-012-9102-9.

Kuusela, M.; Vatanen, T.; Malmi, E.; Raiko, T.; Aaltonen, T.; and Nagai, Y. 2011. Semisupervised anomaly detection - towards model independent searches of new physics. arXiv: 1102.3329v1[physics.data-an] 14 Dec 2011.

Legg, S., and Hutter, M. 2007. Universal Intelligence: A Definition of Machine Intelligence. Minds and Machines 17(4):391-444.

Leitgeb, H., and Ladyman, J. 2008. Criteria of Identity and Structuralist Ontology. Philosophia Mathematica III(16):388-396.

Macbeth, D. 2011. Seeing how it goes: Paper-and-pencil reasoning in mathematical practice. Philosophia Mathematica. DOI:10.1093phimat/nkr006.

Maddy, P. 2007. Second Philosophy: A Naturalistic Method. Oxford: Oxford University Press.

Mittelstaedt, P. 2011. The problem of interpretation of modern physics. Foundation of Physics. DOI:10.1007/10701-011-9571-2.

Moran, D. 2008. Husserl's Transcendental Philosophy and the Critique of Naturalism. Cont. Philos. Rev. doi: 10.1007/s11007-008-9088-3.

Pollock, J. L. 2006. Against Optimality: Logical Foundations for Decision-Task Planning in Autonomous Agents. Computational Intelligence 20(1):1-25.

Schlosshauer, M. 2008. Classicality, the Ensemble Interpretation, and Decoherence: Resolving the Hyperion Dispute. Found. Phys. doi: 10.1007/s10701-008-9237-x.

Schmid, U., and Kitzelmann, E. 2011. Inductive rule learning on the knowledge level.

Schreiber, L., and Sommer, H. 2010. Can Mathematics be Justified by Natural Logic? In Dubois, D., ed., Computing Anticipatory Systems, volume 1303, 189-198. CASYS'09 Ninth International Conference.

Sher, G. 2010. Is logic in the mind or in the world? Synthese. doi: 10.1007/S11229-010-9796-1. 
Sommer, H. 2009. Emergence of Classical Reality From a Quantum Mechanical Background. Chaos, Solitons and Fractals 39:1027-1036.

Srivastava, S.; Immerman, N.; and Zilberstein, S. 2010. A New Representation and Associated Algorithms for Generalized Planning. Artificial Intelligence. doi: 10.1016/j.artint.2010.10.006.

Stenlund, S. 2010. Different senses of finitude: An inquiry into Hilbert's finitism. Synthese. doi: 10.1007/S11229-010-9823-2.

Stuckey, W.; Silberstein, M.; and Cifone, M. 2009. Reconciling Spacetime and the Quantum: Relational Blockworld and the Quantum Liar Paradox. Found. Phys. 38:348-383. doi: 10.1007/s10701-008-9206-4.

Symons, J. 2008. Computational Models of Emergent Properties. Minds and Machines. doi: 10.1007/s11023-008-9120-8.

Tarrazo, M. 2004. Schopenhauer's prolegomenon to fuzziness. Fuzzy Optimization and Decision Making 3:227-254.

Tversky, A., and Kahnemann, D. 1983. Extension versus intuitive reasoning: the conjunction fallacy in probabiliy judgment. Psychological Review 90(4):293-315.

Veness, J.; Ng, K.; Hutter, M.; and Silver, D. 2009. A Monte Carlo AIXI Approximation. arXiv: 0909.0801v1[cs.AI]4Sep,2009.

Wang, P. 1994. From inheritance relations to nonaxiomatic logic. Int. J. of approximate Reasoning 11:281-319.

Wang, P. 2007. Three Fundamental Misconceptions of Artificial Intelligence. J. Experimental and Theoretical Artificial Intelligence 19(3):249-268.

Wang, P. 2009. Formalization of Evidence: Comparative Study. J. Artificial General Intelligence $1: 25-53$.

Wang, P. 2011. From NARS to a thinking machine. http://www.cis.temple.edu/ppwang/.

Weyl, H. 1968. Gesammelte Werke, volume 3. Berlin, Heidelberg, New York: Springer. chapter The Gost of Modality, 684-709.

Yager, R. 1994. Aggregation operators and fuzzy systems modeling. Fuzzy Sets and Systems 67(2):129-145.

Zeh, H. 1999. The Meaning of Decoherence. arXiv: quant-ph/9905004v2 29.6.1999.

Zuh, D. 2011. Wogegen wandte sich Husserl 1891? Husserl Stud. doi: 10.1007/s10743-11-9100-3.

Zurek, W. 1998. Relative States and the Environment: Einselection, and the Existential Interpretation. Philos. Trans. R. Soc. Lond. Ser.A 356:1793-1821. arXiv: [quant-ph]9805065.

Zurek, W. 2007. Relative States and the Environment: Einselection, Envariance, Quantum Darwinismus, and the Existential Interpretation. arXiv: 0707.2832v1[quant-ph]19.7.2007. 